Proceedings of the Edinburgh Mathematical Society (2007) 50,653-671 (C)

DOI:10.1017/S0013091505001823 Printed in the United Kingdom

\title{
INTERPOLATION METHODS DEFINED BY MEANS OF POLYGONS AND COMPACT OPERATORS
}

\author{
LUZ M. FERNÁNDEZ-CABRERA ${ }^{1}$ AND ANTÓN MARTÍNEZ ${ }^{2}$ \\ ${ }^{1}$ Sección Departamental de Matemática Aplicada, \\ Escuela de Estadística, Universidad Complutense de Madrid, \\ 28040 Madrid, Spain (luz_fernandez-c@mat.ucm.es) \\ ${ }^{2}$ Departamento de Matemática Aplicada I, \\ Escuela Técnica Superior Ingenieros Industriales, \\ Universidad de Vigo, 36200 Vigo, \\ Spain (antonmar@uvigo.es)
}

(Received 12 December 2005)

\begin{abstract}
We work with interpolation methods for $N$-tuples of Banach spaces associated with polygons. We compare necessary conditions for interpolating closed operator ideals with conditions required to interpolate compactness. We also establish a formula for the measure of non-compactness of interpolated operators.
\end{abstract}

Keywords: interpolation methods associated with polygons; compact operators; closed operator ideals; measure of non-compactness

2000 Mathematics subject classification: Primary 46B70; 47B10

\section{Introduction}

The study of interpolation methods for finite families ( $N$-tuples) of Banach spaces has been of interest since the beginning of abstract interpolation theory in the early 1960s. The first contributions on this problem were due to Foiaş and Lions [19], Yoshikawa [25], Favini [16], Sparr [23] and Fernandez [17]; other references can be found in the monographs by Triebel [24] and Brudnyĩ and Krugljak [1]. More recently, in 1991, Cobos and Peetre [4] introduced interpolation methods for $N$-tuples of Banach spaces associated with a convex polygon $\Pi$ in the plane and a point $(\alpha, \beta)$ in the interior of $\Pi$. Using the vertices of $\Pi$ they defined $K$ - and $J$-functionals with two parameters and then they introduced $K$ - and $J$-spaces by means of an $(\alpha, \beta)$-weighted $L_{q}$-norm. (We shall recall the definitions in $\S 2$.) In particular, when $\Pi$ is equal to the simplex, these methods yield (the first non-trivial case of) spaces introduced by Sparr [23], and if $\Pi$ is equal to the unit square, they recover spaces studied by Fernandez [17]. Among other things, the geometrical approach in [4] explains the restriction on parameters in Fernandez's case. 
An important part of [4] is devoted to investigating the behaviour of compact operators in this multi-dimensional case. So, in $[4, \S \S 4-6]$, Cobos and Peetre established compactness results when the interpolated operator acts between two $K$-spaces or two $J$-spaces. Later, Cobos et al. [6] considered operators acting from a $J$-space into a $K$-space. A comparison between the two cases was made in [2]. Norms of interpolated operators acting from a $J$-space into a $K$-space can be estimated by a product of positive powers of the norms of the restrictions of the operator to the Banach spaces forming the $N$-tuple (see (2.2) below), but for operators acting between two $K$-spaces or two $J$-spaces a more involved estimate holds (see (2.1)). This explains the gap between the two cases. Other compactness results can be found in [5].

An estimate for the measure of non-compactness in the situation considered by Cobos et al. was established in [10]. No similar result is known for the case studied by Cobos and Peetre. Accordingly, in the present paper we investigate compactness of operators between two $K$-spaces or two $J$-spaces more thoroughly.

We start by comparing necessary conditions for interpolating compactness with conditions required to interpolate other operator ideals. Towards this aim, we characterize compactness of interpolated operators in terms of a weaker stipulation and a condition depending on the $J$ - or $K$-functionals. In the instance of the real method for couples, the first result of this kind was proved in [18]. Later, it was shown in $[\mathbf{1 1}, \mathbf{1 2}]$ that corresponding results hold for the complex method and other classical methods for couples.

We also establish a formula for the measure of non-compactness of operators acting between two $K$-spaces. This formula is a quantitative version of the corresponding qualitative result of Cobos and Peetre. Finally, as applications of our characterizations and the formula, we recover the results on interpolation of compact operators between general $N$-tuples due to Cobos and Peetre [4].

The plan of the paper is as follows. In $\S 2$ we recall some basic ideas on $K$ - and $J$-spaces associated with polygons. Compactness results for operators interpolated by the $J$-method are established in $\S 3$, and the case for the $K$-method is considered in $\S 4$.

\section{Preliminaries}

Let $\Pi=\overline{P_{1} \cdots P_{N}}$ be a convex polygon in the affine plane $\mathbb{R}^{2}$. The vertices of $\Pi$ are $P_{j}=\left(x_{j}, y_{j}\right), j=1, \ldots, N$. Let $\bar{A}=\left\{A_{1}, \ldots, A_{N}\right\}$ be a Banach $N$-tuple, that is to say, a family of $N$ Banach spaces $A_{j}$, all of which are continuously embedded in a common Hausdorff topological vector space. It is useful to think of $A_{j}$ as sitting on the vertex $P_{j}$. Using the polygon $\Pi$, we define the $K$ - and $J$-functionals by

$$
\begin{aligned}
& K(t, s ; a)=\inf \left\{\sum_{j=1}^{N} t^{x_{j}} s^{y_{j}}\left\|a_{j}\right\|_{A_{j}}: a=\sum_{j=1}^{N} a_{j}, a_{j} \in A_{j}\right\}, \\
& J(t, s ; a)=\max \left\{t^{x_{j}} s^{y_{j}}\|a\|_{A_{j}}: 1 \leqslant j \leqslant N\right\} .
\end{aligned}
$$

Here $t, s>0$. It is easy to check that $\{K(t, s ; \cdot)\}_{t, s>0}$ is a family of norms on $\Sigma(\bar{A})=A_{1}+\cdots+A_{N}$, with any two of them being equivalent. Similarly, $\{J(t, s ; \cdot)\}_{t, s>0}$ 
is a family of norms on $\Delta(\bar{A})=A_{1} \cap \cdots \cap A_{N}$, with any two of them being equivalent. Note that $K(1,1 ; \cdot)$ coincides with the norm of $\Sigma(\bar{A})$ and $J(1,1 ; \cdot)=\|\cdot\|_{\Delta(\bar{A})}$.

Let $1 \leqslant q \leqslant \infty$ and let $(\alpha, \beta)$ be any point in the interior of $\Pi,(\alpha, \beta) \in$ Int $\Pi$. The $K$-space $\bar{A}_{(\alpha, \beta), q ; K}$ is formed by all elements $a \in \Sigma(\bar{A})$ having a finite norm

$$
\|a\|_{\bar{A}_{(\alpha, \beta), q ; K}}=\left(\sum_{(m, n) \in \mathbb{Z}^{2}}\left(2^{-\alpha m-\beta n} K\left(2^{m}, 2^{n} ; a\right)\right)^{q}\right)^{1 / q}
$$

(the sum should be replaced by the supremum if $q=\infty$ ). The $J$-space $\bar{A}_{(\alpha, \beta), q ; J}$ is formed by all those $a \in \Sigma(\bar{A})$ that can be represented as

$$
\left.a=\sum_{(m, n) \in \mathbb{Z}^{2}} u_{m, n} \quad \text { (convergence in } \Sigma(\bar{A})\right)
$$

with $\left\{u_{m, n}\right\} \subseteq \Delta(\bar{A})$ and

$$
\left(\sum_{(m, n) \in \mathbb{Z}^{2}}\left(2^{-\alpha m-\beta n} J\left(2^{m}, 2^{n} ; u_{m, n}\right)\right)^{q}\right)^{1 / q}<\infty
$$

(again the sum should be replaced by the supremum if $q=\infty$ ). The norm in $\bar{A}_{(\alpha, \beta), q ; J}$ is

$$
\|a\|_{\bar{A}_{(\alpha, \beta), q ; J}}=\inf \left\{\left(\sum_{(m, n) \in \mathbb{Z}^{2}}\left(2^{-\alpha m-\beta n} J\left(2^{m}, 2^{n} ; u_{m, n}\right)\right)^{q}\right)^{1 / q}\right\}
$$

where the infimum is taken over all representations $\left\{u_{m, n}\right\}$, as above.

Spaces $\bar{A}_{(\alpha, \beta), q ; K}$ and $\bar{A}_{(\alpha, \beta), q ; J}$ are the interpolation spaces associated with polygons realized in a discrete way. They were introduced in $[4]$. When $\Pi$ is equal to the simplex $\{(0,0),(1,0),(0,1)\}$, the spaces coincide with (the first non-trivial case of) spaces investigated by Sparr [23]. If $\Pi$ is the unit square $\{(0,0),(1,0),(1,1),(0,1)\}$, we recover spaces studied by Fernandez [17].

$K$ - and $J$-spaces do not coincide in general $[\mathbf{7}, \mathbf{1 3}, \mathbf{2 3}]$. We have only that $\bar{A}_{(\alpha, \beta), q ; J} \hookrightarrow$ $\bar{A}_{(\alpha, \beta), q ; K}$, where ' $\hookrightarrow$ ' denotes continuous inclusion. Let us give a concrete example. Let $\Pi$ be the unit square and let $\ell_{1}\left(w_{n}\right)$ be the weighted $\ell_{1}$-space with weights $\left\{w_{n}\right\}$. It is shown in [7, Example 2.8] that

$$
\left(\ell_{1}\left(\frac{1}{\sqrt{n}}\right), \ell_{1}\left(\frac{1}{n}\right), \ell_{1}\left(\frac{1}{\sqrt{n}}\right), \ell_{1}\left(\frac{1}{n}\right)\right)_{\left(\frac{1}{2}, \frac{1}{2}\right), 1 ; J}=\ell_{1}\left(\frac{1}{\sqrt{n}}\right)
$$

and

$$
\left(\ell_{1}\left(\frac{1}{\sqrt{n}}\right), \ell_{1}\left(\frac{1}{n}\right), \ell_{1}\left(\frac{1}{\sqrt{n}}\right), \ell_{1}\left(\frac{1}{n}\right)\right)_{\left(\frac{1}{2}, \frac{1}{2}\right), 1 ; K}=\ell_{1}\left(\frac{1+\log n}{n}\right)
$$

Other interpolation formulae will be mentioned later on (see (3.2) and (4.2)). More examples can be found in, for example, $[\mathbf{3}, \mathbf{4}, \mathbf{7}]$. 


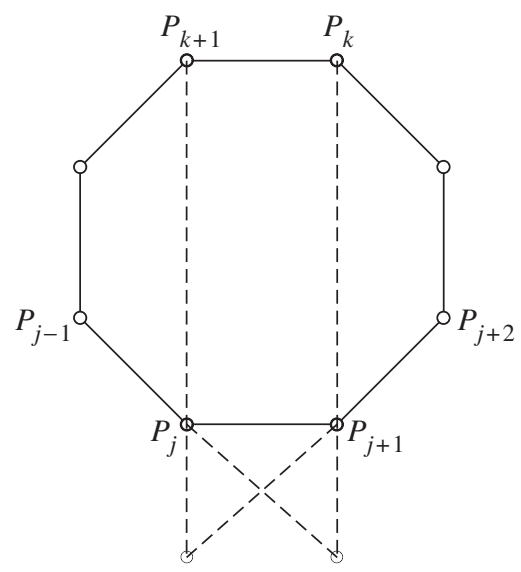

Figure 1. Admissible polygon.

Let $\bar{B}=\left\{B_{1}, \ldots, B_{N}\right\}$ be another Banach $N$-tuple. We write $T \in \mathcal{L}(\bar{A}, \bar{B})$ to mean that $T$ is a linear operator from $\Sigma(\bar{A})$ to $\Sigma(\bar{B})$ whose restriction to each $A_{j}$ defines a bounded operator from $A_{j}$ into $B_{j}, j=1, \ldots, N$.

If $T \in \mathcal{L}(\bar{A}, \bar{B})$, it is not difficult to check that the restriction of $T$ to the $K$-space defines a bounded operator $T: \bar{A}_{(\alpha, \beta), q ; K} \rightarrow \bar{B}_{(\alpha, \beta), q ; K}$. The same holds for $J$-spaces. As for norm estimates, it was shown in [7, Theorem 1.9] that

$$
\|T\|_{\bar{A}_{(\alpha, \beta), q ; K}, \bar{B}_{(\alpha, \beta), q ; K}} \leqslant C \max \left\{\|T\|_{A_{i}, B_{i}}^{c_{i}}\|T\|_{A_{r}, B_{r}}^{c_{r}}\|T\|_{A_{s}, B_{s}}^{c_{s}}:\{i, r, s\} \in \mathcal{P}_{\alpha, \beta}\right\} .
$$

Here $\mathcal{P}_{\alpha, \beta}$ is the set of all triples $\{i, r, s\}$ such that $(\alpha, \beta)$ belongs to the triangle with vertices $P_{i}, P_{r}, P_{s}$, numbers $c_{i}, c_{r}, c_{s}$ are the (unique) baricentric coordinates of $(\alpha, \beta)$ with respect to $P_{i}, P_{r}, P_{s}$, and $C$ is a constant depending only on $\Pi$ and $(\alpha, \beta)$. A similar inequality holds for the restriction of $T$ to the $J$-spaces.

For operators acting from a $J$-space into a $K$-space, a different estimate holds. Let $\bar{\theta}=\left(\theta_{1}, \ldots, \theta_{N}\right)$ be any $N$-tuple of positive numbers with $\sum_{j=1}^{N} \theta_{j}=1$ and $\sum_{j=1}^{N} \theta_{j} P_{j}=$ $(\alpha, \beta)$. It is shown in [7, Theorem 3.2] that there is a constant $M>0$, depending only on $\bar{\theta}$, such that, for any Banach $N$-tuples $\bar{A}=\left\{A_{1}, \ldots, A_{N}\right\}, \bar{B}=\left\{B_{1}, \ldots, B_{N}\right\}$ and any $T \in \mathcal{L}(\bar{A}, \bar{B})$, we have

$$
\|T\|_{\bar{A}_{(\alpha, \beta), q ; J}, \bar{B}_{(\alpha, \beta), q ; K}} \leqslant M \prod_{j=1}^{N}\|T\|_{A_{j}, B_{j}}^{\theta_{j}} .
$$

The following class of polygons was introduced in [4]. If $\Pi=\overline{P_{1} \cdots P_{N}}$, then for $j>N$ or $j<1$, we put

$$
P_{j}=P_{j_{0}} \quad \text { if } j \equiv j_{0}(\bmod N), 1 \leqslant j_{0} \leqslant N .
$$

Definition 2.1. The convex polygon $\Pi$ (see Figure 1) is said to be admissible if, for each edge $\overline{P_{j} P_{j+1}}, j=1, \ldots, N$, there is another $\overline{P_{k} P_{k+1}}$ satisfying the following two conditions. 
(a) The extension of the segment $\overline{P_{j} P_{k+1}}$ in the direction of $P_{j}$ meets the extension of $\overline{P_{j+1} P_{j+2}}$ in the direction of $P_{j+1}$.

(b) The extension of the segment $\overline{P_{j+1} P_{k}}$ in the direction of $P_{j+1}$ meets the extension of $\overline{P_{j-1} P_{j}}$ in the direction of $P_{j}$.

It is clear that any regular polygon with at least five edges is admissible.

The compactness results of Cobos and Peetre for general $N$-tuples require that $\Pi$ is the simplex, the unit square or any admissible polygon $[4, \S 6]$. Interpolation results for weakly compact operators and other closed operator ideals do not require admissibility of the polygon but require that $1<q<\infty[\mathbf{8}, \mathbf{9}]$. Next we review some concepts from operator theory.

Let $E, F$ be Banach spaces. We designate by $\mathcal{L}(E, F)$ the collection of all bounded linear operators from $E$ into $F$, endowed with the usual operator norm. We put $U_{E}$ for the closed unit ball of $E$, and $E^{*}$ for the dual space of $E$.

We write $\ell_{1}\left(U_{E}\right)$ for the Banach space of all absolutely summable families of scalars $\left\{\lambda_{x}\right\}_{x \in U_{E}}$ with $U_{E}$ as the index set. We write $\ell_{\infty}\left(U_{F^{*}}\right)$ for the Banach space of all bounded families of scalars indexed by the elements of $U_{F^{*}}$. The operators $\iota_{F}: F \rightarrow$ $\ell_{\infty}\left(U_{F^{*}}\right), \pi_{E}: \ell_{1}\left(U_{E}\right) \rightarrow E$ defined by $\iota_{F} y=\{\langle f, y\rangle\}_{f \in U_{F^{*}}}$ and $\pi_{E}\left\{\lambda_{x}\right\}=\sum_{x \in U_{E}} \lambda_{x} x$ are an isometric embedding and a metric surjection, respectively.

An operator ideal $\mathcal{J}$ is a method of ascribing to each pair $(E, F)$ of Banach spaces a linear subspace $\mathcal{J}(E, F)$ of $\mathcal{L}(E, F)$ such that $\mathcal{J}(E, F)$ contains the finite rank operators, and it satisfies the condition that $S T R \in \mathcal{J}(G, V)$ whenever $R \in \mathcal{L}(G, E), T \in \mathcal{J}(E, F)$ and $S \in \mathcal{L}(F, V)$.

The operator ideal $\mathcal{J}$ is said to be closed if $\mathcal{J}(E, F)$ is a closed subspace of $\mathcal{L}(E, F)$ for all Banach spaces $E$ and $F$. The ideal $\mathcal{J}$ is said to be injective (respectively, surjective) if, whenever $T \in \mathcal{L}(E, F)$ and, in addition, $\iota_{F} T \in \mathcal{J}\left(E, \ell_{\infty}\left(U_{F^{*}}\right)\right)$ (respectively, $\left.T \pi_{E} \in \mathcal{J}\left(\ell_{1}\left(U_{E}\right), F\right)\right)$, it follows that $T \in \mathcal{J}(E, F)$. Compact operators and weakly compact operators are examples of surjective injective closed operator ideals. Strictly singular operators form an ideal which is closed and injective but is not surjective, while the ideal of strictly cosingular operators is closed and surjective but is not injective. (We refer the reader to $[\mathbf{1 4}, \mathbf{2 1}]$ for more details on operator ideals.)

Given any double sequence of Banach spaces $\left\{W_{m, n}\right\}_{(m, n) \in \mathbb{Z}^{2}}$ and any sequence of non-negative numbers $\left\{\lambda_{m, n}\right\}_{(m, n) \in \mathbb{Z}^{2}}$ we put

$$
\begin{aligned}
\ell_{q}\left(\lambda_{m, n} W_{m, n}\right)=\left\{w=\left\{w_{m, n}\right\}: w_{m, n} \in W_{m, n}\right. \text { and } & \\
& \left.\|w\|_{\ell_{q}\left(\lambda_{m, n} W_{m, n}\right)}=\left(\sum_{(m, n) \in \mathbb{Z}^{2}}\left(\lambda_{m, n}\left\|w_{m, n}\right\|_{W_{m, n}}\right)^{q}\right)^{1 / q}<\infty\right\} .
\end{aligned}
$$

When $\lambda_{m, n}=1$ for all $(m, n) \in \mathbb{Z}^{2}$, we write simply $\ell_{q}\left(W_{m, n}\right)$. Let $O_{r, s}: \ell_{q}\left(W_{m, n}\right) \rightarrow$ $W_{r, s}$ be the projection $O_{r, s}\left\{w_{m, n}\right\}=w_{r, s}$, and let $D_{r, s}: W_{r, s} \rightarrow \ell_{q}\left(W_{m, n}\right)$ be the embedding $D_{r, s} w=\left\{\delta_{m, n}^{r, s} w\right\}$, where $\delta_{m, n}^{r, s}$ is the Kronecker delta.

Let $1<q<\infty$. An operator ideal $\mathcal{J}$ is said to satisfy the $\Sigma_{q}$-condition if, for any sequences of Banach spaces $\left\{W_{m, n}\right\},\left\{V_{m, n}\right\}$ and for any operator $T \in$ 
$\mathcal{L}\left(\ell_{q}\left(W_{m, n}\right), \ell_{q}\left(V_{m, n}\right)\right)$, it follows from $O_{u, v} T D_{r, s} \in \mathcal{J}\left(W_{r, s}, V_{u, v}\right)$ for any $r, s, u, v \in \mathbb{Z}$ that $T \in \mathcal{J}\left(\ell_{q}\left(W_{m, n}\right), \ell_{q}\left(V_{m, n}\right)\right)$. For example, weakly compact operators, Rosenthal operators, Banach-Saks operators and dual Radon-Nikodým operators satisfy the $\Sigma_{q}$-condition [20]. On the other hand, it is clear that compact operators fail the $\Sigma_{q}$-condition.

\section{Compactness and $J$-spaces}

Given any convex polygon $\Pi=\overline{P_{1} \cdots P_{N}}$ with $P_{j}=\left(x_{j}, y_{j}\right)$ and any Banach $N$-tuple $\bar{A}=\left\{A_{1}, \ldots, A_{N}\right\}$, we denote by $G_{m, n}$ the Banach space $\Delta(\bar{A})$ endowed with the norm $J\left(2^{m}, 2^{n} ; \cdot\right)$. We write

$$
\ell_{1}(j)=\ell_{1}\left(2^{-x_{j} m-y_{j} n} G_{m, n}\right), \quad j=1, \ldots, N, \quad \text { and } \quad \bar{\ell}_{1}=\left\{\ell_{1}(1), \ldots, \ell_{1}(N)\right\} .
$$

According to [4, Theorem 3.1], if $(\alpha, \beta) \in \operatorname{Int} \Pi$ and $1 \leqslant q \leqslant \infty$, we have

$$
\left(\bar{\ell}_{1}\right)_{(\alpha, \beta), q ; J}=\ell_{q}\left(2^{-\alpha m-\beta n} G_{m, n}\right) \quad \text { (equivalent norms). }
$$

Let $\pi$ be the operator defined by $\pi\left\{u_{m, n}\right\}=\sum_{m, n \in \mathbb{Z}^{2}} u_{m, n}$. It is easy to check that $\pi \in \mathcal{L}\left(\ell_{1}(j), A_{j}\right), j=1, \ldots, N$. Moreover, $\pi$ acting from $\ell_{q}\left(2^{-\alpha m-\beta n} G_{m, n}\right)$ into $\bar{A}_{(\alpha, \beta), q ; J}$ is a metric surjection.

The following result shows a necessary and sufficient condition for the interpolated operator by the $J$-method to belong to a surjective closed operator ideal. It complements $[\mathbf{9}$, Theorem 2.6] and [8, Theorem 3.2].

Theorem 3.1. Let $\Pi=\overline{P_{1} \cdots P_{N}}$ be a convex polygon in $\mathbb{R}^{2}$ with vertices $P_{j}=$ $\left(x_{j}, y_{j}\right)$, let $(\alpha, \beta) \in \operatorname{Int} \Pi, 1<q<\infty$, and let $\mathcal{J}$ be a surjective closed operator ideal which satisfies the $\Sigma_{q}$-condition. Assume that $\bar{A}=\left\{A_{1}, \ldots, A_{N}\right\}$ and $\bar{B}=\left\{B_{1}, \ldots, B_{N}\right\}$ are Banach $N$-tuples and let $T \in \mathcal{L}(\bar{A}, \bar{B})$.

Then a necessary and sufficient condition for $T: \bar{A}_{(\alpha, \beta), q ; J} \rightarrow \bar{B}_{(\alpha, \beta), q ; J}$ to belong to J is that $T: \Delta(\bar{A}) \rightarrow \bar{B}_{(\alpha, \beta), q ; J}$ belongs to J.

Proof. The factorization

$$
\Delta(\bar{A}) \hookrightarrow \bar{A}_{(\alpha, \beta), q ; J} \stackrel{T}{\rightarrow} \bar{B}_{(\alpha, \beta), q ; J}
$$

shows that if $T \in \mathcal{J}\left(\bar{A}_{(\alpha, \beta), q ; J}, \bar{B}_{(\alpha, \beta), q ; J}\right)$ then $T: \Delta(\bar{A}) \rightarrow \bar{B}_{(\alpha, \beta), q ; J}$ belongs to J.

Conversely, if $T \in \mathcal{J}\left(\Delta(\bar{A}), \bar{B}_{(\alpha, \beta), q ; J}\right)$, then $T \pi D_{r, s} \in \mathcal{J}\left(2^{-\alpha r-\beta s} G_{r, s}, \bar{B}_{(\alpha, \beta), q ; J}\right)$ for any $(r, s) \in \mathbb{Z}^{2}$. Here $\left\{D_{r, s}\right\}_{(r, s) \in \mathbb{Z}^{2}}$ are the operators introduced in $\S 2$. Since J satisfies the $\Sigma_{q}$-property, it follows that $T \pi \in \mathcal{J}\left(\ell_{q}\left(2^{-\alpha m-\beta n} G_{m, n}\right), \bar{B}_{(\alpha, \beta), q ; J}\right)$. Then, using the fact that $\mathcal{J}$ is surjective, we conclude that $T: \bar{A}_{(\alpha, \beta), q ; J} \rightarrow \bar{B}_{(\alpha, \beta), q ; J}$ belongs to J.

Theorem 3.1 does not apply to compact operators because they do not satisfy the $\Sigma_{q}$-condition. In fact, a similar equivalence does not hold for compact operators, as we show next by means of an example. 
Example 3.2. Let $\Pi=\{(0,0),(1,0),(1,1),(0,1)\}$ be the unit square; take any $(\alpha, \beta) \in \operatorname{Int} \Pi$ and let

$$
\bar{A}=\left\{\ell_{1}, \ell_{1}\left(2^{-m}\right), \ell_{1}\left(2^{-m-n}\right), \ell_{1}\left(2^{-n}\right)\right\}, \quad \bar{B}=\left\{\ell_{\infty}, \ell_{\infty}\left(2^{-m}\right), \ell_{\infty}\left(2^{-m-n}\right), \ell_{\infty}\left(2^{-n}\right)\right\} .
$$

Choose $T \in \mathcal{L}(\bar{A}, \bar{B})$ as the operator defined by $T\left\{\xi_{m, n}\right\}=\left\{\mu_{m, n}\right\}$, where

$$
\mu_{m, n}= \begin{cases}\xi_{m, n} & \text { if } m \geqslant 0 \text { and } n \geqslant 0, \\ 0 & \text { otherwise. }\end{cases}
$$

According to [4, Corollary 3.2] we have

$$
\bar{A}_{(\alpha, \beta), 2 ; J}=\bar{B}_{(\alpha, \beta), 2 ; J}=\ell_{2}\left(2^{-\alpha m-\beta n}\right) .
$$

On the other hand, a direct computation shows that

$$
\Delta(\bar{A})=\ell_{1}\left(\max \left\{1,2^{-m}, 2^{-m-n}, 2^{-n}\right\}\right) .
$$

So, $T: \bar{A}_{(\alpha, \beta), 2 ; J} \rightarrow \bar{B}_{(\alpha, \beta), 2 ; J}$ is not compact, but $T: \Delta(\bar{A}) \rightarrow \bar{B}_{(\alpha, \beta), 2 ; J}$ is compact because it is the limit of the sequence of finite rank operators $\left\{T_{r}\right\}_{r \in \mathbb{N}}$ defined by $T_{r}\left\{\xi_{m, n}\right\}=\left\{\rho_{m, n}\right\}$ with

$$
\rho_{m, n}= \begin{cases}\xi_{m, n} & \text { if } 0 \leqslant m \leqslant r \text { and } 0 \leqslant n \leqslant r \\ 0 & \text { otherwise. }\end{cases}
$$

Next we investigate when compactness of $T: \Delta(\bar{A}) \rightarrow \bar{B}_{(\alpha, \beta), q ; J}$ passes to

$$
T: \bar{A}_{(\alpha, \beta), q ; J} \rightarrow \bar{B}_{(\alpha, \beta), q ; J} .
$$

First we recall a construction of Cobos and Peetre for the simplex, the unit square or any admissible polygon $\Pi=\overline{P_{1} \cdots P_{N}}[4, \S 5]$.

There is a family $\left\{\Omega_{\nu}^{(r)}\right\}_{0 \leqslant r \leqslant N, \nu \in \mathbb{N}}$ of subsets of $\mathbb{R}^{2}$ such that

(i) for each $\nu \in \mathbb{N},\left\{\Omega_{\nu}^{(0)}, \Omega_{\nu}^{(1)}, \ldots, \Omega_{\nu}^{(N)}\right\}$ is a partition of $\mathbb{R}^{2}$,

(ii) the sets $\left\{\Omega_{\nu}^{(0)}\right\}_{\nu \in \mathbb{N}}$ are bounded, with $\Omega_{1}^{(0)} \subseteq \Omega_{2}^{(0)} \subseteq \cdots$, and, given any bounded set $K \subseteq \mathbb{R}^{2}$, there exists a $\nu \in \mathbb{N}$ such that $K \subseteq \Omega_{\nu}^{(\overline{0)}}$,

(iii) for any $1 \leqslant j \leqslant N$, we have $\Omega_{1}^{(j)} \supseteq \Omega_{2}^{(j)} \supseteq \cdots$.

Moreover, if $\bar{A}=\left\{A_{1}, \ldots, A_{N}\right\}$ is any Banach $N$-tuple and we define the $N$-tuple $\bar{\ell}_{1}$ as in (3.1), then the family $\left\{Q_{\nu}^{(r)}\right\}_{0 \leqslant r \leqslant N, \nu \in \mathbb{N}}$ of projections on $\bar{\ell}_{1}$ defined by $Q_{\nu}^{(r)}\left\{u_{m, n}\right\}=$ $\left\{w_{m, n}\right\}$, where

$$
w_{m, n}= \begin{cases}u_{m, n} & \text { if }(m, n) \in \Omega_{\nu}^{(r)} \\ 0 & \text { otherwise }\end{cases}
$$

satisfy the following conditions. 
(I) The identity operator $I$ on $\Sigma\left(\bar{\ell}_{1}\right)$ can be decomposed as

$$
I=\sum_{r=0}^{N} Q_{\nu}^{(r)}, \quad \nu=1,2, \ldots
$$

(II) We have $\left\|Q_{\nu}^{(r)}\right\|_{\ell_{1}(j), \ell_{1}(j)}=1$ for any $\nu \in \mathbb{N}, 0 \leqslant r \leqslant N$ and $1 \leqslant j \leqslant N$.

(III) For each $\nu \in \mathbb{N}, Q_{\nu}^{(0)} \in \mathcal{L}\left(\Sigma\left(\bar{\ell}_{1}\right), \Delta\left(\bar{\ell}_{1}\right)\right)$.

(IV) Let $1 \leqslant j \leqslant N$ and let $1 \leqslant k \leqslant N$ with $k \neq j, j+1$. Then one has either that

(a) for any $\nu \in \mathbb{N}, Q_{\nu}^{(j)}$ maps $\ell_{1}(k)$ to $\ell_{1}(j)$ and the sequence of norms $\left\{\left\|Q_{\nu}^{(j)}\right\|_{\ell_{1}(k), \ell_{1}(j)}\right\}$ converges to 0 as $\nu \rightarrow \infty$, or that

(b) for any $\nu \in \mathbb{N}, Q_{\nu}^{(j)}$ maps $\ell_{1}(k)$ to $\ell_{1}(j+1)$ and the sequence of norms $\left\{\left\|Q_{\nu}^{(j)}\right\|_{\ell_{1}(k), \ell_{1}(j+1)}\right\}$ converges to 0 as $\nu \rightarrow \infty$.

In fact, it is shown in $[\mathbf{4}$, Theorem 5.5] that

$$
\begin{aligned}
& \left\|Q_{\nu}^{(j)}\right\|_{\ell_{1}(k), \ell_{1}(j)} \leqslant 2^{-\nu} \text { for case (a), } \\
& \left\|Q_{\nu}^{(j)}\right\|_{\ell_{1}(k), \ell_{1}(j+1)} \leqslant 2^{-\nu} \text { for case (b). }
\end{aligned}
$$

Now we are prepared to characterize compactness of $T: \bar{A}_{(\alpha, \beta), q ; J} \rightarrow \bar{B}_{(\alpha, \beta), q ; J}$ in terms of $T: \Delta(\bar{A}) \rightarrow \bar{B}_{(\alpha, \beta), q ; J}$.

Theorem 3.3. Let $\Pi=\overline{P_{1} \cdots P_{N}}$ be the simplex, the unit square or any admissible polygon with vertices $P_{j}=\left(x_{j}, y_{j}\right)$, let $(\alpha, \beta) \in \operatorname{Int} \Pi$ and $1 \leqslant q \leqslant \infty$. Assume that $\bar{A}=\left\{A_{1}, \ldots, A_{N}\right\}$ and $\bar{B}=\left\{B_{1}, \ldots, B_{N}\right\}$ are Banach $N$-tuples, and that $T \in \mathcal{L}(\bar{A}, \bar{B})$. Then $T: \bar{A}_{(\alpha, \beta), q ; J} \rightarrow \bar{B}_{(\alpha, \beta), q ; J}$ is compact if and only if the following conditions hold:

(a) $T: \Delta(\bar{A}) \rightarrow \bar{B}_{(\alpha, \beta), q ; J}$ is compact;

(b) $\sup \left\{\left\|T\left(\sum_{|m|>k,|n|>k} u_{m, n}\right)\right\|_{\bar{B}_{(\alpha, \beta), q ; J}}:\left\|\left\{u_{m, n}\right\}\right\|_{\ell_{q}\left(2^{-\alpha m-\beta n} G_{m, n}\right)} \leqslant 1\right\} \rightarrow 0$ as $k \rightarrow \infty$.

Proof. First note that, by (ii), condition (b) is equivalent to

$$
\sup \left\{\left\|T\left(\sum_{(m, n) \notin \Omega_{\nu}^{(0)}} u_{m, n}\right)\right\|_{\bar{B}_{(\alpha, \beta), q ; J}}:\left\|\left\{u_{m, n}\right\}\right\|_{\ell_{q}\left(2^{-\alpha m-\beta n} G_{m, n}\right)} \leqslant 1\right\} \rightarrow 0
$$

as $\nu \rightarrow \infty$. That is to say, (b) is equivalent to the following.

$\left(\mathrm{b}^{\prime}\right)\left\|T \pi-T \pi Q_{\nu}^{(0)}\right\|_{\ell_{q}\left(2^{-\alpha m-\beta n} G_{m, n}\right), \bar{B}_{(\alpha, \beta), q ; J}} \rightarrow 0 \quad$ as $\nu \rightarrow \infty$. 
Suppose that (a) and $\left(\mathrm{b}^{\prime}\right)$ are satisfied. Using (III), we can factorize

$$
T \pi Q_{\nu}^{(0)}: \ell_{q}\left(2^{-\alpha m-\beta n} G_{m, n}\right) \rightarrow \bar{B}_{(\alpha, \beta), q ; J}
$$

as

$$
\ell_{q}\left(2^{-\alpha m-\beta n} G_{m, n}\right) \stackrel{Q_{\nu}^{(0)}}{\longrightarrow} \Delta\left(\bar{\ell}_{1}\right) \stackrel{\pi}{\rightarrow} \Delta(\bar{A}) \stackrel{T}{\rightarrow} \bar{B}_{(\alpha, \beta), q ; J} .
$$

Hence, (a) implies that $T \pi Q_{\nu}^{(0)}$ is compact for any $\nu \in \mathbb{N}$. By $\left(\mathrm{b}^{\prime}\right)$, it follows that $T \pi$ : $\ell_{q}\left(2^{-\alpha m-\beta n} G_{m, n}\right) \rightarrow \bar{B}_{(\alpha, \beta), q ; J}$ is compact. Since $\pi$ is a metric surjection, we conclude that $T: \bar{A}_{(\alpha, \beta), q ; J} \rightarrow \bar{B}_{(\alpha, \beta), q ; J}$ is compact.

Conversely, if $T: \bar{A}_{(\alpha, \beta), q ; J} \rightarrow \bar{B}_{(\alpha, \beta), q ; J}$ is compact, then (a) follows from the embed$\operatorname{ding} \Delta(\bar{A}) \hookrightarrow \bar{A}_{(\alpha, \beta), q ; J}$. In order to establish $\left(\mathrm{b}^{\prime}\right)$, observe that

$$
\left(I-Q_{\nu}^{(0)}\right) \circ\left(I-Q_{\nu+1}^{(0)}\right)=I-Q_{\nu+1}^{(0)}, \quad \nu \in \mathbb{N} .
$$

Thus, the sequence

$$
\left\{\left\|T \pi\left(I-Q_{\nu}^{(0)}\right)\right\|_{\ell_{q}\left(2^{-\alpha m-\beta n} G_{m, n}\right), \bar{B}_{(\alpha, \beta), q ; J}}\right\}
$$

is non-increasing. Let $\delta$ be its limit. We need to show that $\delta=0$. Find $\left\{w_{\nu}\right\} \subseteq$ $\left.U_{\ell_{q}(2-\alpha m-\beta n} G_{m, n}\right)$ such that

$$
\delta=\lim _{\nu \rightarrow \infty}\left\|T \pi\left(I-Q_{\nu}^{(0)}\right) w_{\nu}\right\|_{\bar{B}_{(\alpha, \beta), q ; J}} .
$$

The sequence $\left\{\pi\left(I-Q_{\nu}^{(0)}\right) w_{\nu}\right\}$ is contained in $U_{\bar{A}_{(\alpha, \beta), q ; J}}$ because

$$
\left\|I-Q_{\nu}^{(0)}\right\|_{\ell_{q}\left(2^{-\alpha m-\beta n} G_{m, n}\right), \ell_{q}\left(2^{-\alpha m-\beta n} G_{m, n}\right)} \leqslant 1
$$

By the compactness of $T$, we may suppose, passing to a subsequence if necessary, that $\left\{T \pi\left(I-Q_{\nu^{\prime}}^{(0)}\right) w_{\nu^{\prime}}\right\}$ converges to some $b$ in $\bar{B}_{(\alpha, \beta), q ; J}$. Therefore, $\|b\|_{\bar{B}_{(\alpha, \beta), q ; J}}=\delta$.

Note that, by (I),

$$
T \pi\left(I-Q_{\nu^{\prime}}^{(0)}\right) w_{\nu^{\prime}}=\sum_{j=1}^{N} T \pi Q_{\nu^{\prime}}^{(j)} w_{\nu^{\prime}} .
$$

We shall show that $b=0$, and therefore $\delta=0$, by establishing that $\left\{T \pi Q_{\nu^{\prime}}^{(j)} w_{\nu^{\prime}}\right\} \rightarrow 0$ in $\Sigma(\bar{B})$ for $j=1, \ldots, N$.

Fix $1 \leqslant j \leqslant N$ and choose any $1 \leqslant k \leqslant N$ with $k \neq j, j+1$. By (IV), either $Q_{\nu}^{(j)}: \ell_{1}(k) \rightarrow \ell_{1}(j)$ for any $\nu \in \mathbb{N}$ and their norms tend to 0 as $\nu \rightarrow \infty$, or $Q_{\nu}^{(j)}$ : $\ell_{1}(k) \rightarrow \ell_{1}(j+1)$ for any $\nu \in \mathbb{N}$ and $\left\|Q_{\nu}^{(j)}\right\|_{\ell_{1}(k), \ell_{1}(j+1)} \rightarrow 0$ as $\nu \rightarrow \infty$. It follows from the interpolation formula (3.2) and the estimate $(2.2)$ that $Q_{\nu^{\prime}}^{(j)} \operatorname{maps} \ell_{q}\left(2^{-\alpha m-\beta n} G_{m, n}\right)$ to $\ell_{1}(j)+\ell_{1}(j+1)$ with

$$
\left\|Q_{\nu^{\prime}}^{(j)}\right\|_{\ell_{q}\left(2^{-\alpha m-\beta n} G_{m, n}\right), \ell_{1}(j)+\ell_{1}(j+1)} \rightarrow 0 \quad \text { as } \nu^{\prime} \rightarrow \infty
$$

This yields that $\left\{T \pi Q_{\nu^{\prime}}^{(j)} w_{\nu^{\prime}}\right\}$ is a null sequence in $\Sigma(\bar{B})$ and completes the proof.

Next we shall use Theorem 3.3 to derive the following result due to Cobos and Peetre [4, Theorem 6.1]. 
Corollary 3.4. Let $\Pi$ be the simplex, the unit square or any admissible polygon, let $(\alpha, \beta) \in \operatorname{Int} \Pi$ and $1 \leqslant q \leqslant \infty$. Assume that $\bar{A}=\left\{A_{1}, \ldots, A_{N}\right\}$ and $\bar{B}=\left\{B_{1}, \ldots, B_{N}\right\}$ are Banach $N$-tuples, and let $T \in \mathcal{L}(\bar{A}, \bar{B})$ such that $T: A_{j} \rightarrow B_{j}$ is compact for every $1 \leqslant j \leqslant N$. Then

$$
T: \bar{A}_{(\alpha, \beta), q ; J} \rightarrow \bar{B}_{(\alpha, \beta), q ; J}
$$

is compact.

Proof. Since $T: A_{j} \rightarrow B_{j}$ compactly, $T: \Delta(\bar{A}) \rightarrow B_{j}$ is compact for $j=1, \ldots, N$. Applying a Lions-Peetre-type compactness result due to Cobos and Peetre [4, Theorem 4.3], we find that Theorem 3.3 (a) holds. Let us show that ( $\mathrm{b}^{\prime}$ ) also holds. Using (I), we have

$$
T \pi-T \pi Q_{\nu}^{(0)}=\sum_{j=1}^{N} T \pi Q_{\nu}^{(j)}
$$

Hence,

$$
\left\|T \pi-T \pi Q_{\nu}^{(0)}\right\|_{\ell_{q}\left(2^{-\alpha m-\beta n} G_{m, n}\right), \bar{B}_{(\alpha, \beta), q ; J}} \leqslant \sum_{j=1}^{N}\left\|T \pi Q_{\nu}^{(j)}\right\|_{\ell_{q}\left(2^{-\alpha m-\beta n} G_{m, n}\right), \bar{B}_{(\alpha, \beta), q ; J}} .
$$

Take any $1 \leqslant j \leqslant N$; let us check that

$$
\left\|T \pi Q_{\nu}^{(j)}\right\|_{\ell_{q}\left(2^{-\alpha m-\beta n} G_{m, n}\right), \bar{B}_{(\alpha, \beta), q ; J}} \rightarrow 0 \quad \text { as } \nu \rightarrow \infty .
$$

By (3.2) and the norm estimate (2.1), we have

$$
\begin{aligned}
& \left\|T \pi Q_{\nu}^{(j)}\right\|_{\ell_{q}\left(2^{-\alpha m-\beta n} G_{m, n}\right), \bar{B}_{(\alpha, \beta), q ; J}} \\
& \quad \leqslant C \max _{\{i, r, s\} \in \mathcal{P}_{\alpha, \beta}}\left\{\left\|T \pi Q_{\nu}^{(j)}\right\|_{\ell_{1}(i), B_{i}}^{c_{i}}\left\|T \pi Q_{\nu}^{(j)}\right\|_{\ell_{1}(r), B_{r}}^{c_{r}}\left\|T \pi Q_{\nu}^{(j)}\right\|_{\ell_{1}(s), B_{s}}^{c_{s}}\right\} .
\end{aligned}
$$

Therefore, in order to establish (3.3) it suffices to prove that, for any $1 \leqslant k \leqslant N$ with $k \neq j, j+1$, we have

$$
\left\|T \pi Q_{\nu}^{(j)}\right\|_{\ell_{1}(k), B_{k}} \rightarrow 0 \quad \text { as } \nu \rightarrow \infty .
$$

Since $Q_{\nu-1}^{(j)} \circ Q_{\nu}^{(j)}=Q_{\nu}^{(j)}$, the sequence $\left\{\left\|T \pi Q_{\nu}^{(j)}\right\|_{\ell_{1}(k), B_{k}}\right\}$ is non-increasing. Let $\mu$ be its limit. Choose a sequence $\left\{u_{\nu}\right\} \subseteq U_{\ell_{1}(k)}$ such that $\mu=\lim _{\nu \rightarrow \infty}\left\|T \pi Q_{\nu}^{(j)} u_{\nu}\right\|_{B_{k}}$. By (II), the sequence $\left\{\pi Q_{\nu}^{(j)} u_{\nu}\right\}$ is bounded in $A_{k}$. Using the compactness of $T: A_{k} \rightarrow B_{k}$, we can find a subsequence $\left\{T \pi Q_{\nu^{\prime}}^{(j)} u_{\nu^{\prime}}\right\}$ converging to some $b$ in $B_{k}$. Therefore, $\mu=\|b\|_{B_{k}}$. Again, it follows by (IV) that $\left\{T \pi Q_{\nu^{\prime}}^{(j)} u_{\nu^{\prime}}\right\}$ converges to 0 in $\Sigma(\bar{B})$. Consequently, $b=0$ and so $\mu=0$. This establishes (3.4) and completes the proof.

\section{Compactness and $K$-spaces}

In this section we investigate operators interpolated by the $K$-method. If $\Pi=\overline{P_{1} \cdots P_{N}}$ is a convex polygon with vertices $P_{j}=\left(x_{j}, y_{j}\right)$ and $\bar{B}=\left\{B_{1}, \ldots, B_{N}\right\}$ is a Banach $N$-tuple, we denote by $F_{m, n}$ the Banach space $\Sigma(\bar{B})$ provided with the norm $K\left(2^{m}, 2^{n} ; \cdot\right)$. We put

$$
\ell_{\infty}(j)=\ell_{\infty}\left(2^{-x_{j} m-y_{j} n} F_{m, n}\right), \quad j=1, \ldots, N, \quad \text { and } \quad \bar{\ell}_{\infty}=\left\{\ell_{\infty}(1), \ldots, \ell_{\infty}(N)\right\} .
$$


Given any $(\alpha, \beta) \in \operatorname{Int} \Pi$ and $1 \leqslant q \leqslant \infty$, it follows from [4, Theorem 3.1] that

$$
\left(\bar{\ell}_{\infty}\right)_{(\alpha, \beta), q ; K}=\ell_{q}\left(2^{-\alpha m-\beta n} F_{m, n}\right) \quad \text { (equivalent norms) }
$$

Let $\iota$ be the operator assigning to each $b \in \Sigma(\bar{B})$ the constant sequence $\iota b=$ $(\ldots, b, b, b, \ldots)$. Clearly, $\iota \in \mathcal{L}\left(B_{j}, \ell_{\infty}(j)\right)$ for $j=1, \ldots, N$. It is also immediate that $\iota$ acting from $\bar{B}_{(\alpha, \beta), q ; K}$ into $\ell_{q}\left(2^{-\alpha m-\beta n} F_{m, n}\right)$ is an isometric embedding.

We start by characterizing those interpolated operators which belong to a given injective closed operator ideal satisfying the $\Sigma_{q}$-condition. The result complements $[\mathbf{9}$, Theorem 2.6] and [8, Theorem 3.1].

Theorem 4.1. Let $\Pi=\overline{P_{1} \cdots P_{N}}$ be a convex polygon in $\mathbb{R}^{2}$ with vertices $P_{j}=$ $\left(x_{j}, y_{j}\right)$, let $(\alpha, \beta) \in \operatorname{Int} \Pi, 1<q<\infty$ and let $\mathcal{J}$ be an injective closed operator ideal which satisfies the $\Sigma_{q}$-condition. Assume that $\bar{A}=\left\{A_{1}, \ldots, A_{N}\right\}$ and $\bar{B}=\left\{B_{1}, \ldots, B_{N}\right\}$ are Banach $N$-tuples and let $T \in \mathcal{L}(\bar{A}, \bar{B})$.

Then a necessary and sufficient condition for $T: \bar{A}_{(\alpha, \beta), q ; K} \rightarrow \bar{B}_{(\alpha, \beta), q ; K}$ to belong to $\mathcal{J}$ is that $T: \bar{A}_{(\alpha, \beta), q ; K} \rightarrow \Sigma(\bar{B})$ belongs to J.

Proof. Since $\bar{B}_{(\alpha, \beta), q ; K} \hookrightarrow \Sigma(\bar{B})$, if $T \in \mathcal{J}\left(\bar{A}_{(\alpha, \beta), q ; K}, \bar{B}_{(\alpha, \beta), q ; K}\right)$, it follows that $T \in$ $\mathcal{J}\left(\bar{A}_{(\alpha, \beta), q ; K}, \Sigma(\bar{B})\right)$.

Conversely, if $T \in \mathcal{J}\left(\bar{A}_{(\alpha, \beta), q ; K}, \Sigma(\bar{B})\right)$, then for all $(r, s) \in \mathbb{Z}^{2}$ we have that $O_{r, s} \iota T$ belongs to $\mathcal{J}\left(\bar{A}_{(\alpha, \beta), q ; K}, 2^{-\alpha r-\beta s} F_{r, s}\right)$. Here $\left\{O_{r, s}\right\}$ are the operators introduced in the definition of $\Sigma_{q}$-condition (see $\S 2$ ). Since $\mathcal{J}$ satisfies the $\Sigma_{q}$-property, we derive that $\iota T: \bar{A}_{(\alpha, \beta), q ; K} \rightarrow \ell_{q}\left(2^{-\alpha m-\beta n} F_{m, n}\right)$ belongs to J. Finally, injectivity of $\mathcal{J}$ implies that

$$
T \in \mathcal{J}\left(\bar{A}_{(\alpha, \beta), q ; K}, \bar{B}_{(\alpha, \beta), q ; K}\right) .
$$

Working with the unit square, $N$-tuples

$$
\bar{A}=\left\{\ell_{1}, \ell_{1}\left(2^{-m}\right), \ell_{1}\left(2^{-m-n}\right), \ell_{1}\left(2^{-n}\right)\right\}, \quad \bar{B}=\left\{\ell_{\infty}, \ell_{\infty}\left(2^{-m}\right), \ell_{\infty}\left(2^{-m-n}\right), \ell_{\infty}\left(2^{-n}\right)\right\}
$$

and the same operator as in Example 3.2, it is not difficult to show that compactness of $T: \bar{A}_{(\alpha, \beta), q ; K} \rightarrow \Sigma(\bar{B})$ is not sufficient to yield that $T: \bar{A}_{(\alpha, \beta), q ; K} \rightarrow \bar{B}_{(\alpha, \beta), q ; K}$ is compact. Indeed, in this case we have $\Sigma(\bar{B})=\ell_{\infty}\left(\min \left\{1,2^{-m}, 2^{-m-n}, 2^{-n}\right\}\right)$ and, by [4, Corollary 3.2],

$$
\bar{A}_{(\alpha, \beta), 2 ; K}=\bar{B}_{(\alpha, \beta), 2 ; K}=\ell_{2}\left(2^{-\alpha m-\beta n}\right) .
$$

Therefore, if $T$ is as in Example 3.2, we have that $T: \bar{A}_{(\alpha, \beta), 2 ; K} \rightarrow \Sigma(\bar{B})$ is compact but $T: \bar{A}_{(\alpha, \beta), 2 ; K} \rightarrow \bar{B}_{(\alpha, \beta), 2 ; K}$ fails to be compact.

In order to determine compactness of operators interpolated by the $K$-method, let $\Pi$ be the simplex, the unit square or any admissible polygon and let $\left\{\Omega_{\nu}^{(r)}\right\}_{0 \leqslant r \leqslant N, \nu \in \mathbb{N}}$ be the family of subsets of $\mathbb{R}^{2}$ described in $\S 3$. We introduce a new family of subsets by putting

$$
\tilde{\Omega}_{\nu}^{(r)}=\left\{(m, n) \in \mathbb{R}^{2}:(-m,-n) \in \Omega_{\nu}^{(r)}\right\} .
$$


Given any Banach $N$-tuple $\bar{B}=\left\{B_{1}, \ldots, B_{N}\right\}$, we consider the family of projections $\left\{R_{\nu}^{(r)}\right\}_{0 \leqslant r \leqslant N, \nu \in \mathbb{N}}$ on $\bar{\ell}_{\infty}$ defined by

$$
R_{\nu}^{(r)}\left\{v_{m, n}\right\}=\left\{w_{m, n}\right\}, \quad \text { where } w_{m, n}= \begin{cases}v_{m, n} & \text { if }(m, n) \in \tilde{\Omega}_{\nu}^{(r)}, \\ 0 & \text { otherwise. }\end{cases}
$$

These projections satisfy the following properties.

$\left(\mathrm{I}^{\prime}\right)$ The identity operator $I$ on $\Sigma\left(\bar{\ell}_{\infty}\right)$ can be written as

$$
I=\sum_{r=0}^{N} R_{\nu}^{(r)}, \quad \nu=1,2, \ldots
$$

(II') Projections are uniformly bounded in $\bar{\ell}_{\infty}$,

$$
\left\|R_{\nu}^{(r)}\right\|_{\ell_{\infty}(j), \ell_{\infty}(j)}=1 \quad \text { for any } \nu \in \mathbb{N}, 0 \leqslant r \leqslant N \text { and } 1 \leqslant j \leqslant N .
$$

$\left(\mathrm{III}^{\prime}\right)$ For each $\nu \in \mathbb{N}, R_{\nu}^{(0)} \in \mathcal{L}\left(\Sigma\left(\bar{\ell}_{\infty}\right), \Delta\left(\bar{\ell}_{\infty}\right)\right)$.

$\left(\mathrm{IV}^{\prime}\right)$ Let $1 \leqslant j \leqslant N$ and let $1 \leqslant k \leqslant N$ with $k \neq j, j+1$. Then one has that either

(a) for any $\nu \in \mathbb{N}, R_{\nu}^{(j)}$ maps $\ell_{\infty}(j)$ to $\ell_{\infty}(k)$ and the sequence of norms $\left\{\left\|R_{\nu}^{(j)}\right\|_{\ell_{\infty}(j), \ell_{\infty}(k)}\right\}$ converges to 0 as $\nu \rightarrow \infty$, or

(b) for any $\nu \in \mathbb{N}, R_{\nu}^{(j)}$ maps $\ell_{\infty}(j+1)$ to $\ell_{\infty}(k)$ and the sequence of norms $\left\{\left\|R_{\nu}^{(j)}\right\|_{\ell_{\infty}(j+1), \ell_{\infty}(k)}\right\}$ converges to 0 as $\nu \rightarrow \infty$.

To check property $\left(\mathrm{IV}^{\prime}\right)$ observe that if

$$
\left\langle(m, n), P_{k}-P_{j}\right\rangle \leqslant-\nu \quad \text { for all }(m, n) \in \Omega_{\nu}^{(j)},
$$

then

$$
\left\langle(m, n), P_{j}-P_{k}\right\rangle \leqslant-\nu \quad \text { for all }(m, n) \in \tilde{\Omega}_{\nu}^{(j)} .
$$

Here $\langle\cdot, \cdot\rangle$ denotes the scalar product of $\mathbb{R}^{2}$. The other properties are trivially fulfilled.

Theorem 4.2. Let $\Pi=\overline{P_{1} \cdots P_{N}}$ be the simplex, the unit square or any admissible polygon with vertices $P_{j}=\left(x_{j}, y_{j}\right)$, let $(\alpha, \beta) \in \operatorname{Int} \Pi$ and $1 \leqslant q \leqslant \infty$. Assume that $\bar{A}=\left\{A_{1}, \ldots, A_{N}\right\}$ and $\bar{B}=\left\{B_{1}, \ldots, B_{N}\right\}$ are Banach $N$-tuples, and that $T \in \mathcal{L}(\bar{A}, \bar{B})$.

If

(a) $T: \bar{A}_{(\alpha, \beta), q ; K} \rightarrow \Sigma(\bar{B})$ is compact, and

(b) $\sup \left\{\left(\sum_{|m|>k,|n|>k}\left(2^{-\alpha m-\beta n} K\left(2^{m}, 2^{n} ; T a\right)\right)^{q}\right)^{1 / q}:\|a\|_{\bar{A}_{(\alpha, \beta), q ; K}} \leqslant 1\right\} \rightarrow 0$ as $k \rightarrow \infty$, 
then

$$
T: \bar{A}_{(\alpha, \beta), q ; K} \rightarrow \bar{B}_{(\alpha, \beta), q ; K}
$$

is compact.

Moreover, if $q<\infty$, then compactness of $T: \bar{A}_{(\alpha, \beta), q ; K} \rightarrow \bar{B}_{(\alpha, \beta), q ; K}$ implies conditions (a) and (b).

Proof. According to (ii) and the definition of $\tilde{\Omega}_{\nu}^{(0)}$, we observe that the sets $\left\{\tilde{\Omega}_{\nu}^{(0)}\right\}$ are bounded, with $\tilde{\Omega}_{1}^{(0)} \subseteq \tilde{\Omega}_{2}^{(0)} \subseteq \cdots$ and, given any bounded set $K \subseteq \mathbb{R}^{2}$, there exists $\nu \in \mathbb{N}$ such that $K \subseteq \tilde{\Omega}_{\nu}^{(\overline{0})}$. Therefore, condition (b) is equivalent to the following.

$\left(\mathrm{b}^{\prime}\right)\left\|\iota T-R_{\nu}^{(0)} \iota T\right\|_{\bar{A}_{(\alpha, \beta), q ; K}, \ell_{q}\left(2^{-\alpha m-\beta n} F_{m, n}\right)} \rightarrow 0$ as $\nu \rightarrow \infty$.

By (III'), the operator $R_{\nu}^{(0)} \iota T$ can be factorized as

$$
\bar{A}_{(\alpha, \beta), q ; K} \stackrel{T}{\rightarrow} \Sigma(\bar{B}) \stackrel{\iota}{\rightarrow} \Sigma\left(\bar{\ell}_{\infty}\right) \stackrel{R_{\nu}^{(0)}}{\longrightarrow} \Delta\left(\bar{\ell}_{\infty}\right) \hookrightarrow \ell_{q}\left(2^{-\alpha m-\beta n} F_{m, n}\right) .
$$

Hence, condition (a) implies that, for each $\nu \in \mathbb{N}$, the operator

$$
R_{\nu}^{(0)} \iota T: \bar{A}_{(\alpha, \beta), q ; K} \rightarrow \ell_{q}\left(2^{-\alpha m-\beta n} F_{m, n}\right)
$$

is compact. Using $\left(\mathrm{b}^{\prime}\right)$, we obtain $\iota T: \bar{A}_{(\alpha, \beta), q ; K} \rightarrow \ell_{q}\left(2^{-\alpha m-\beta n} F_{m, n}\right)$ compactly. Since $\iota$ is an isometric embedding, we conclude that $T: \bar{A}_{(\alpha, \beta), q ; K} \rightarrow \bar{B}_{(\alpha, \beta), q ; K}$ is compact.

Assume now that $q<\infty$ and $T: \bar{A}_{(\alpha, \beta), q ; K} \rightarrow \bar{B}_{(\alpha, \beta), q ; K}$ is compact. Condition (a) follows from the embedding $\bar{B}_{(\alpha, \beta), q ; K} \hookrightarrow \Sigma(\bar{B})$. In order to establish $\left(\mathrm{b}^{\prime}\right)$ take any $\varepsilon>0$. By compactness of $T$, there exists a finite set $\left\{a_{1}, \ldots, a_{p}\right\} \subseteq \bar{A}_{(\alpha, \beta), q ; K}$ such that

$$
T\left(U_{\bar{A}_{(\alpha, \beta), q ; K}}\right) \subseteq \bigcup_{s=1}^{p}\left\{T a_{s}+\frac{1}{3} \varepsilon U_{\bar{B}_{(\alpha, \beta), q ; K}}\right\} .
$$

Properties of the sets $\tilde{\Omega}_{\nu}^{(0)}$, and so of the operators $R_{\nu}^{(0)}$, and the assumption that $q<\infty$ yields that there exists a $\nu_{0} \in \mathbb{N}$ such that if $\nu \geqslant \nu_{0}$, then

$$
\left\|\iota T a_{s}-R_{\nu}^{(0)} \iota T a_{s}\right\|_{\ell_{q}\left(2^{-\alpha m-\beta n} F_{m, n}\right)} \leqslant \frac{1}{3} \varepsilon, \quad s=1, \ldots, p .
$$

Consequently, for all $\nu \geqslant \nu_{0}$, given any $a \in U_{\bar{A}_{(\alpha, \beta), q ; K}}$, if we choose $s$ such that

$$
\left\|T a-T a_{s}\right\|_{\bar{B}_{(\alpha, \beta), q ; K}} \leqslant \frac{1}{3} \varepsilon
$$

we obtain

$$
\begin{aligned}
& \left\|\iota T a-R_{\nu}^{(0)} \iota T a\right\|_{\ell_{q}\left(2^{-\alpha m-\beta n} F_{m, n}\right)} \\
& \leqslant\left\|\iota T a-\iota T a_{s}\right\|_{\ell_{q}\left(2^{-\alpha m-\beta n} F_{m, n}\right)}+\left\|\iota T a_{s}-R_{\nu}^{(0)} \iota T a_{s}\right\|_{\ell_{q}\left(2^{-\alpha m-\beta n} F_{m, n}\right)} \\
& +\left\|R_{\nu}^{(0)} \iota T a_{s}-R_{\nu}^{(0)} \iota T a\right\|_{\ell_{q}\left(2^{-\alpha m-\beta n} F_{m, n}\right)} \\
& \leqslant 2\left\|T a-T a_{s}\right\|_{\bar{B}_{(\alpha, \beta), q ; K}}+\frac{1}{3} \varepsilon \\
& \leqslant \varepsilon \text {. }
\end{aligned}
$$

This gives $\left(b^{\prime}\right)$ and completes the proof. 
Next we establish a formula for the measure of non-compactness of an operator interpolated by the $K$-method. Recall that if $T \in \mathcal{L}(A, B)$, the measure of non-compactness $\beta(T)=\beta\left(T_{A, B}\right)$ of $T$ is defined as the infimum of all $\sigma>0$ such that there exists a finite number of elements $b_{1}, \ldots, b_{s} \in B$ such that

$$
T\left(U_{A}\right) \subseteq \bigcup_{j=1}^{s}\left\{b_{j}+\sigma U_{B}\right\} .
$$

Clearly, $\beta\left(T_{A, B}\right) \leqslant\|T\|_{A, B}$, and $T$ is compact if and only if $\beta(T)=0$. Other properties of this notion can be found in $[\mathbf{1 5}, \mathbf{2 2}]$.

Given any Banach $N$-tuple $\bar{A}=\left\{A_{1}, \ldots, A_{N}\right\}$, we denote by $\bar{A}^{\circ}=\left\{A_{1}^{\circ}, \ldots, A_{N}^{\circ}\right\}$ the Banach $N$-tuple formed by the closures of $\Delta(\bar{A})$ in $A_{j}$ for $1 \leqslant j \leqslant N$. We say that $\bar{A}$ is regular if $A_{j}^{\circ}=A_{j}$ for $j=1, \ldots, N$.

Theorem 4.3. Let $\Pi=\overline{P_{1} \cdots P_{N}}$ be the simplex, the unit square or any admissible polygon with vertices $P_{j}=\left(x_{j}, y_{j}\right)$, let $(\alpha, \beta) \in \operatorname{Int} \Pi$ and $1 \leqslant q \leqslant \infty$. Let $\bar{A}=$ $\left\{A_{1}, \ldots, A_{N}\right\}$ be a regular Banach $N$-tuple, let $\bar{B}=\left\{B_{1}, \ldots, B_{N}\right\}$ be a Banach $N$-tuple and assume that $T \in \mathcal{L}(\bar{A}, \bar{B})$. Then

$$
\beta\left(T_{\bar{A}_{(\alpha, \beta), q ; K}, \bar{B}_{(\alpha, \beta), q ; K}}\right) \leqslant M \sum_{j=1}^{N} \max _{\{i, r, s\} \in \mathcal{P}_{\alpha, \beta}}\left\{\eta_{j, i}(T)^{c_{i}} \eta_{j, r}(T)^{c_{r}} \eta_{j, s}(T)^{c_{s}}\right\} .
$$

Here

$$
\eta_{j, k}(T)= \begin{cases}\beta\left(T_{A_{k}, B_{k}}\right) & \text { if } k \neq j, j+1, \\ \|T\|_{A_{k}, B_{k}} & \text { if } k=j, j+1,\end{cases}
$$

and $M$ is a constant depending only on $\Pi$ and $(\alpha, \beta)$.

Proof. Using $\left(\mathrm{I}^{\prime}\right)$ and the fact that $\iota: \bar{B}_{(\alpha, \beta), q ; K} \rightarrow \ell_{q}\left(2^{-\alpha m-\beta n} F_{m, n}\right)$ is an isometric embedding, we have

$\beta\left(T_{\bar{A}_{(\alpha, \beta), q ; K}, \bar{B}_{(\alpha, \beta), q ; K}}\right) \leqslant 2 \beta\left(\iota T_{\bar{A}_{(\alpha, \beta), q ; K}, \ell_{q}\left(2^{-\alpha m-\beta n} F_{m, n}\right)}\right) \leqslant 2 \beta\left(R_{\nu}^{(0)} \iota T\right)+2 \sum_{j=1}^{N} \beta\left(R_{\nu}^{(j)} \iota T\right)$,

where we consider all operators $R_{\nu}^{(r)} \iota T$ acting from $\bar{A}_{(\alpha, \beta), q ; K}$ into $\ell_{q}\left(2^{-\alpha m-\beta n} F_{m, n}\right)$.

Take any $1 \leqslant j \leqslant N$. By [4, Theorem 3.1], the embedding $\left(\bar{\ell}_{\infty}\right)_{(\alpha, \beta), q ; K} \hookrightarrow$ $\ell_{q}\left(2^{-\alpha m-\beta n} F_{m, n}\right)$ has norm less than or equal to 1 . According to the estimate (2.1) for the norms of interpolated operators, we get

$$
\begin{aligned}
& \beta\left(R_{\nu}^{(j)} \iota T\right) \\
& \quad \leqslant\left\|R_{\nu}^{(j)} \iota T\right\|_{\bar{A}_{(\alpha, \beta), q ; K},\left(\bar{\ell}_{\infty}\right)_{(\alpha, \beta), q ; K},} \\
& \quad \leqslant C \max \left\{\left\|R_{\nu}^{(j)} \iota T\right\|_{A_{i}, \ell_{\infty}(i)}^{c_{i}}\left\|R_{\nu}^{(j)} \iota T\right\|_{A_{r}, \ell_{\infty}(r)}^{c_{r}}\left\|R_{\nu}^{(j)} \iota T\right\|_{A_{s}, \ell_{\infty}(s)}^{c_{s}}:\{i, r, s\} \in \mathcal{P}_{\alpha, \beta}\right\} .
\end{aligned}
$$

If $k=j, j+1$, we have

$$
\left\|R_{\nu}^{(j)} \iota T\right\|_{A_{k}, \ell_{\infty}(k)} \leqslant\|T\|_{A_{k}, B_{k}}=\eta_{j, k}(T) .
$$


Assume that $k \neq j, j+1$ and take any $\varepsilon>0$. By the definition of the measure of non-compactness and the fact that $\bar{A}$ is a regular $N$-tuple, there exists a finite set $\left\{a_{1}, \ldots, a_{p}\right\} \subseteq \Delta(\bar{A})$ with $\left\|a_{s}\right\|_{A_{k}} \leqslant 1$ for $s=1, \ldots, p$ and such that

$$
\min \left\{\left\|T a-T a_{s}\right\|_{B_{k}}: 1 \leqslant s \leqslant p\right\} \leqslant 2(1+\varepsilon) \beta\left(T_{A_{k}, B_{k}}\right) \quad \text { for all } a \in U_{A_{k}} .
$$

If we are, say, in the first case of $\left(\mathrm{IV}^{\prime}\right)$, we can find $\nu_{0} \in \mathbb{N}$ such that, for any $\nu \geqslant \nu_{0}$,

$$
\left\|R_{\nu}^{(j)}\right\|_{\ell_{\infty}(j), \ell_{\infty}(k)}\left\|\iota T a_{s}\right\|_{\ell_{\infty}(j)} \leqslant \varepsilon \text { for } s=1, \ldots, p .
$$

Whence, for any $a \in U_{A_{k}}$, choosing $a_{s}$ so that $\left\|T a-T a_{s}\right\|_{B_{k}} \leqslant 2(1+\varepsilon) \beta\left(T_{A_{k}, B_{k}}\right)$, we obtain

$$
\begin{aligned}
\left\|R_{\nu}^{(j)} \iota T a\right\|_{\ell_{\infty}(k)} & \leqslant\left\|R_{\nu}^{(j)} \iota\left(T a-T a_{s}\right)\right\|_{\ell_{\infty}(k)}+\left\|R_{\nu}^{(j)} \iota T a_{s}\right\|_{\ell_{\infty}(k)} \\
& \leqslant 2(1+\varepsilon) \beta\left(T_{A_{k}, B_{k}}\right)+\left\|R_{\nu}^{(j)}\right\|_{\ell_{\infty}(j), \ell_{\infty}(k)}\left\|\iota T a_{s}\right\|_{\ell_{\infty}(j)} \\
& \leqslant 2(1+\varepsilon) \beta\left(T_{A_{k}, B_{k}}\right)+\varepsilon .
\end{aligned}
$$

This implies that

$$
\left\|R_{\nu}^{(j)} \iota T\right\|_{A_{k}, \ell_{\infty}(k)} \leqslant 2 \beta\left(T_{A_{k}, B_{k}}\right)=2 \eta_{j, k}(T) .
$$

Inserting these estimates in (4.4), we get for any $\nu \geqslant \nu_{0}$

$$
\beta\left(R_{\nu}^{(j)} \iota T\right) \leqslant 2 C \max _{\{i, r, s\} \in \mathcal{P}_{\alpha, \beta}}\left\{\eta_{j, i}(T)^{c_{i}} \eta_{j, r}(T)^{c_{r}} \eta_{j, s}(T)^{c_{s}}\right\}, \quad j=1, \ldots, N .
$$

In order to deal with $\beta\left(R_{\nu}^{(0)} \iota T\right)$, take any $\sigma_{j}>\beta\left(T_{A_{j}, B_{j}}\right), j=1, \ldots, N$. It is easy to check that there are Banach spaces $Z_{j}$ and compact linear operators $S_{j} \in \mathcal{L}\left(Z_{j}, B_{j}\right)$ such that

$$
T\left(U_{A_{j}}\right) \subseteq \sigma_{j} U_{B_{j}}+S_{j}\left(U_{Z_{j}}\right), \quad j=1, \ldots, N .
$$

Denote by $\left(Z_{1} \oplus \cdots \oplus Z_{N}\right)_{\ell_{\infty}}$ the direct sum of $Z_{1}, \ldots, Z_{N}$, normed by $\left\|\left(z_{1}, \ldots, z_{N}\right)\right\|=$ $\max \left\{\left\|z_{j}\right\|_{Z_{j}}: 1 \leqslant j \leqslant N\right\}$ and, for each $(m, n) \in \mathbb{Z}^{2}$, write $W_{m, n}=\left(Z_{1} \oplus \cdots \oplus Z_{N}\right)_{\ell_{\infty}}$.

Given any $\varepsilon>0$ and any $(r, s) \in \mathbb{Z}^{2}$, consider the operator $S: \ell_{q}\left(W_{m, n}\right) \rightarrow$ $\ell_{q}\left(2^{-\alpha m-\beta n} F_{m, n}\right)$ defined by $S\left\{w_{m, n}\right\}=\left\{b_{m, n}\right\}$ with

$$
b_{m, n}= \begin{cases}\sum_{j=1}^{N}(1+\varepsilon) 2^{\left(\alpha-x_{j}\right)(m+r)+\left(\beta-y_{j}\right)(n+s)} S_{j} w_{j, m, n} & \text { if }(m, n) \in \tilde{\Omega}_{\nu}^{(0)}, \\ 0 & \text { otherwise, }\end{cases}
$$

where $w_{m, n}=\left(w_{1, m, n}, \ldots, w_{N, m, n}\right)$. The operator $S$ is compact because, using projections from the vector-valued spaces into the factors and using the fact that each $S_{j}$ is compact and that the set $\tilde{\Omega}_{\nu}^{(0)}$ is finite, $S$ can be written as a finite sum of compact operators. We will show that

$$
\begin{aligned}
& R_{\nu}^{(0)} \iota T\left(U_{\bar{A}_{(\alpha, \beta), q ; K}}\right) \\
& \quad \subseteq\left((1+\varepsilon) N \max _{1 \leqslant j \leqslant N}\left\{2^{r\left(\alpha-x_{j}\right)+s\left(\beta-y_{j}\right)} \sigma_{j}\right\}\right) U_{\ell_{q}\left(2^{-\alpha m-\beta n} F_{m, n}\right)}+S\left(U_{\ell_{q}\left(W_{m, n}\right)}\right) .
\end{aligned}
$$


Take any $a \in U_{\bar{A}_{(\alpha, \beta), q ; K}}$ and set

$$
d_{m, n}=d_{m, n}(a)=(1+\varepsilon) 2^{-\alpha m-\beta n} K\left(2^{m}, 2^{n} ; a\right) .
$$

Since $K\left(2^{m+r}, 2^{n+s} ; a\right)<2^{\alpha(m+r)+\beta(n+s)} d_{m+r, n+s}$, we can decompose $a$ in the form $a=$ $\sum_{j=1}^{N} a_{j, m, n}$ with $a_{j, m, n} \in A_{j}$ and

$$
2^{(m+r) x_{j}+(n+s) y_{j}}\left\|a_{j, m, n}\right\|_{A_{j}} \leqslant 2^{\alpha(m+r)+\beta(n+s)} d_{m+r, n+s} .
$$

Then, since

$$
\left\|d_{m+r, n+s}^{-1} 2^{\left(x_{j}-\alpha\right)(m+r)+\left(y_{j}-\beta\right)(n+s)} a_{j, m, n}\right\|_{A_{j}} \leqslant 1,
$$

it follows from (4.6) that there exist $z_{j, m, n} \in U_{Z_{j}}, j=1, \ldots, N$, such that

$$
\begin{aligned}
\| T a_{j, m, n}-S_{j}\left(2^{\left(\alpha-x_{j}\right)(m+r)+\left(\beta-y_{j}\right)(n+s)}\right. & \left.d_{m+r, n+s} z_{j, m, n}\right) \|_{B_{j}} \\
& \leqslant 2^{\left(\alpha-x_{j}\right)(m+r)+\left(\beta-y_{j}\right)(n+s)} \sigma_{j} d_{m+r, n+s} .
\end{aligned}
$$

Let $z=\left\{\left((1+\varepsilon)^{-1} d_{m+r, n+s} z_{j, m, n}\right)_{1 \leqslant j \leqslant N}\right\}_{(m, n) \in \mathbb{Z}^{2}}$. Then

$$
\|z\|_{\ell_{q}\left(W_{m, n}\right)} \leqslant(1+\varepsilon)^{-1}(1+\varepsilon)\left(\sum_{(m, n) \in \mathbb{Z}^{2}}\left(2^{-\alpha m-\beta n} K\left(2^{m}, 2^{n} ; a\right)\right)^{q}\right)^{1 / q} \leqslant 1
$$

and, by (4.8),

$$
\begin{aligned}
& \left\|\left(R_{\nu}^{(0)} \iota T\right) a-S z\right\|_{\ell_{q}\left(2^{-\alpha m-\beta n} F_{m, n}\right)}^{q}\left[2^{-\alpha m-\beta n}\left(\sum_{j=1}^{N} 2^{x_{j} m+y_{j} n}\left\|T a_{j, m, n}-2^{\left(\alpha-x_{j}\right)(m+r)+\left(\beta-y_{j}\right)(n+s)} \times d_{m+r, n+s} S_{j} z_{j, m, n}\right\|_{B_{j}}\right)\right]^{q} \\
& \leqslant \sum_{(m, n) \in \tilde{\Omega}_{\nu}^{(0)}}\left[\sum_{j=1}^{q} 2^{x_{j} m+y_{j} n} 2^{\left(\alpha-x_{j}\right)(m+r)+\left(\beta-y_{j}\right)(n+s)} \sigma_{j} d_{m+r, n+s}\right)^{q} \\
& \leqslant \quad\left(N \max _{1 \leqslant j \leqslant N}\left\{2^{\left(\alpha-x_{j}\right) r+\left(\beta-y_{j}\right) s} \sigma_{j}\right\}\right)^{q} \sum_{(m, n) \in \tilde{\Omega}_{\nu}^{(0)}} d_{m+r, n+s}^{q} \\
& \leqslant(1+\varepsilon)^{q}\left(N \max _{1 \leqslant j \leqslant N}\left\{2^{\left(\alpha-x_{j}\right) r+\left(\beta-y_{j}\right) s} \sigma_{j}\right\}\right)^{q} .
\end{aligned}
$$

This establishes (4.7).

Since $\varepsilon>0$ is arbitrary, we obtain

$$
\beta\left(R_{\nu}^{(0)} \iota T\right) \leqslant N \inf _{(r, s) \in \mathbb{Z}^{2}}\left(\max _{1 \leqslant j \leqslant N}\left\{2^{\left(\alpha-x_{j}\right) r+\left(\beta-y_{j}\right) s} \sigma_{j}\right\}\right) .
$$

Then [7, Theorem 1.9] implies that

$$
\beta\left(R_{\nu}^{(0)} \iota T\right) \leqslant N D \max \left\{\sigma_{i}^{c_{i}} \sigma_{r}^{c_{r}} \sigma_{s}^{c_{s}}:\{i, r, s\} \in \mathcal{P}_{\alpha, \beta}\right\} .
$$


Consequently, for any $\nu \in \mathbb{N}$,

$$
\beta\left(R_{\nu}^{(0)} \iota T\right) \leqslant D \sum_{j=1}^{N} \max \left\{\eta_{j, i}(T)^{c_{i}} \eta_{j, r}(T)^{c_{r}} \eta_{j, s}(T)^{c_{s}}:\{i, r, s\} \in \mathcal{P}_{\alpha, \beta}\right\} .
$$

The last inequality along with (4.5) and (4.3) show that

$$
\beta\left(T_{\bar{A}_{(\alpha, \beta), q ; K}, \bar{B}_{(\alpha, \beta), q ; K}}\right) \leqslant M \sum_{j=1}^{N} \max \left\{\eta_{j, i}(T)^{c_{i}} \eta_{j, r}(T)^{c_{r}} \eta_{j, s}(T)^{c_{s}}:\{i, r, s\} \in \mathcal{P}_{\alpha, \beta}\right\},
$$

where $M=2 D+4 C$.

As an application we obtain the following result of Cobos and Peetre [4].

Corollary 4.4. Let $\Pi$ be the simplex, the unit square or any admissible polygon, let $(\alpha, \beta) \in \operatorname{Int} \Pi$ and $1 \leqslant q \leqslant \infty$. Assume that $\bar{A}=\left\{A_{1}, \ldots, A_{N}\right\}$ and $\bar{B}=\left\{B_{1}, \ldots, B_{N}\right\}$ are Banach $N$-tuples, and let $T \in \mathcal{L}(\bar{A}, \bar{B})$ such that $T: A_{j} \rightarrow B_{j}$ compactly for every $1 \leqslant j \leqslant N$. Then

$$
T: \bar{A}_{(\alpha, \beta), q ; K}^{\circ} \rightarrow \bar{B}_{(\alpha, \beta), q ; K}^{\circ}
$$

is compact.

Proof. For $1 \leqslant j \leqslant N$, the operator $T: A_{j}^{\circ} \rightarrow B_{j}^{\circ}$ is still compact and the $N$-tuple $\bar{A}^{\circ}=\left\{A_{1}^{\circ}, \ldots, A_{N}^{\circ}\right\}$ is regular, so Theorem 4.3 gives the desired result.

Remark 4.5. The proof given by Cobos and Peetre (see [4, Theorem 6.3]) is not entirely correct. They work with the sets $\left\{\Omega_{\nu}^{(r)}\right\}$ and the projections associated with them, but the property they really need on $\left[4\right.$, p. 399] is not $(\mathrm{IV})$, but $\left(\mathrm{IV}^{\prime}\right)$. If one works instead with the projections associated with the sets $\left\{\tilde{\Omega}_{\nu}^{(r)}\right\}$, then their arguments are correct.

We end the paper with a result that characterizes compactness of interpolated operators between $N$-tuples with the property that $J$ - and $K$ - spaces coincide.

Corollary 4.6. Let $\Pi$ be the simplex, the unit square or any admissible polygon, let $(\alpha, \beta) \in \operatorname{Int} \Pi$ and $1 \leqslant q \leqslant \infty$. Assume that $\bar{A}=\left\{A_{1}, \ldots, A_{N}\right\}$ and $\bar{B}=\left\{B_{1}, \ldots, B_{N}\right\}$ are Banach $N$-tuples with $\bar{A}_{(\alpha, \beta), q ; J}=\bar{A}_{(\alpha, \beta), q ; K}$ and $\bar{B}_{(\alpha, \beta), q ; J}=\bar{B}_{(\alpha, \beta), q ; K}$, and let $T \in \mathcal{L}(\bar{A}, \bar{B})$.

If

(a) $T: \Delta(\bar{A}) \rightarrow \Sigma(\bar{B})$ is compact,

(b) $\sup \left\{\left\|T\left(\sum_{|m|>k,|n|>k} u_{m, n}\right)\right\|_{\bar{B}_{(\alpha, \beta), q ; J}}:\left\|\left\{u_{m, n}\right\}\right\|_{\ell_{q}\left(2^{-\alpha m-\beta n} G_{m, n}\right)} \leqslant 1\right\} \rightarrow 0$ as $k \rightarrow \infty$,

(c) $\sup \left\{\left(\sum_{|m|>k,|n|>k}\left(2^{-\alpha m-\beta n} K\left(2^{m}, 2^{n} ; T a\right)\right)^{q}\right)^{1 / q}:\|a\|_{\bar{A}_{(\alpha, \beta), q ; K}} \leqslant 1\right\} \rightarrow 0$ as $k \rightarrow \infty$, 
then

(d) $T: \bar{A}_{(\alpha, \beta), q ; K} \rightarrow \bar{B}_{(\alpha, \beta), q ; J}$ is compact.

Furthermore, if $q<\infty$, then (d) implies (a), (b) and (c).

Proof. Assume first that $1 \leqslant q<\infty$ and that (d) holds. Then (a) is a consequence of the factorization

$$
\Delta(\bar{A}) \hookrightarrow \bar{A}_{(\alpha, \beta), q ; K} \stackrel{T}{\rightarrow} \bar{B}_{(\alpha, \beta), q ; J} \hookrightarrow \Sigma(\bar{B}) .
$$

Moreover, since restrictions

$$
T: \bar{A}_{(\alpha, \beta), q ; J} \rightarrow \bar{B}_{(\alpha, \beta), q ; J}, \quad T: \bar{A}_{(\alpha, \beta), q ; K} \rightarrow \bar{B}_{(\alpha, \beta), q ; K}
$$

are compact as well, (b) and (c) follow from Theorems 3.3 and 4.2.

Conversely, if $1 \leqslant q \leqslant \infty$ and (a)-(c) are satisfied, then, using the diagram

$$
\ell_{q}\left(2^{-\alpha m-\beta n} G_{m, n}\right) \stackrel{Q_{\nu}^{(0)}}{\longrightarrow} \Delta\left(\bar{\ell}_{1}\right) \stackrel{\pi}{\rightarrow} \Delta(\bar{A}) \stackrel{T}{\longrightarrow} \Sigma(\bar{B}) \stackrel{\iota}{\rightarrow} \Sigma\left(\bar{\ell}_{\infty}\right) \stackrel{R_{\nu}^{(0)}}{\longrightarrow} \ell_{q}\left(2^{-\alpha m-\beta n} F_{m, n}\right),
$$

we find that $R_{\nu}^{(0)} \iota T \pi Q_{\nu}^{(0)}$ is compact for each $\nu \in \mathbb{N}$. We claim that

$$
\left\{R_{\nu}^{(0)} \iota T \pi Q_{\nu}^{(0)}\right\} \rightarrow \iota T \pi \quad \text { in } \mathcal{L}\left(\ell_{q}\left(2^{-\alpha m-\beta n} G_{m, n}\right), \ell_{q}\left(2^{-\alpha m-\beta n} F_{m, n}\right)\right) .
$$

Indeed, by conditions (b) and (c), we obtain

$$
\begin{aligned}
& \left\|\iota T \pi-R_{\nu}^{(0)} \iota T \pi Q_{\nu}^{(0)}\right\|_{\ell_{q}\left(2^{-\alpha m-\beta n} G_{m, n}\right), \ell_{q}\left(2^{-\alpha m-\beta n} F_{m, n}\right)} \\
& \leqslant C \\
& \quad+C\left(I-R_{\nu}^{(0)}\right) \iota T \|_{\bar{A}_{(\alpha, \beta), q ; K}, \ell_{q}\left(2^{-\alpha m-\beta n} F_{m, n}\right)} \\
& \quad+C\left\|T \pi\left(I-Q_{\nu}^{(0)}\right)\right\|_{\ell_{q}\left(2^{-\alpha m-\beta n} G_{m, n}\right), \bar{B}_{(\alpha, \beta), q ; J}} \rightarrow 0 \quad \text { as } \nu \rightarrow \infty .
\end{aligned}
$$

This implies that $\iota T \pi: \ell_{q}\left(2^{-\alpha m-\beta n} G_{m, n}\right) \rightarrow \ell_{q}\left(2^{-\alpha m-\beta n} F_{m, n}\right)$ is compact, and therefore $T: \bar{A}_{(\alpha, \beta), q ; J} \rightarrow \bar{B}_{(\alpha, \beta), q ; K}$ compactly. Finally, since $\bar{A}_{(\alpha, \beta), q ; J}=\bar{A}_{(\alpha, \beta), q ; K}$ and $\bar{B}_{(\alpha, \beta), q ; J}=\bar{B}_{(\alpha, \beta), q ; K}$, we derive that $T: \bar{A}_{(\alpha, \beta), q ; K} \rightarrow \bar{B}_{(\alpha, \beta), q ; J}$ is compact.

Acknowledgements. The authors have been supported in part by the Spanish Ministerio de Educación y Ciencia (MTM2004-01888) and by the CAM-UCM (Grupo de Investigación 910348).

\section{References}

1. Y. BRUdNYİ AND N. KRUGLJAK, Interpolation functors and interpolation spaces, Volume 1 (North-Holland, Amsterdam, 1991).

2. F. Совоs, On the optimality of compactness results for interpolation methods associated to polygons, Indagationes Math. 5 (1994), 397-401.

3. F. COBOS AND J. MARTín, On interpolation of function spaces by methods defined by polygons, J. Approx. Theory 132 (2005), 182-203.

4. F. Cobos And J. Peetre, Interpolation of compact operators: the multidimensional case, Proc. Lond. Math. Soc. 63 (1991), 371-400. 
5. F. Cobos And R. Romero, Lions-Peetre type compactness results for several Banach spaces, Math. Inequal. Applic. 7 (2004), 557-571.

6. F. Cobos, T. Kühn And T. SchonbeK, One-sided compactness results for AronszajnGagliardo functors, J. Funct. Analysis 106 (1992), 274-313.

7. F. Cobos, P. Fernández-Martínez And T. SchonbeK, Norm estimates for interpolation methods defined by means of polygons, J. Approx. Theory 80 (1995), 321-351.

8. F. Cobos, J. M. Cordeiro and A. Martínez, Quantitative estimates for interpolated operators by multidimensional methods, Rev. Mat. Complut. 12 (1999), 85-103.

9. F. Cobos, P. Fernández-Martínez And A. Martínez, On reiteration and the behaviour of weak compactness under certain interpolation methods, Collect. Math. 50 (1999), 53-72.

10. F. Cobos, P. Fernández-Martínez and A. Martínez, Measure of non-compactness and interpolation methods associated to polygons, Glasgow Math. J. 41 (1999), 65-79.

11. F. Cobos, L. M. Fernández-Cabrera And A. Martínez, Complex interpolation, minimal methods and compact operators, Math. Nachr. 264 (2004), 67-82.

12. F. Cobos, L. M. Fernández-Cabrera And A. Martínez, Compact operators between $K$ - and $J$-spaces, Studia Math. 166 (2005), 199-220.

13. M. CWIKel And S. Janson, Real and complex interpolation methods for finite and infinite families of Banach spaces, Adv. Math. 66 (1987), 234-290.

14. J. Diestel, H. Jarchow And A. TONGE, Absolutely summing operators, Cambridge Studies in Advanced Mathematics, Volume 43 (Cambridge University Press, 1995).

15. D. E. Edmunds And W. D. Evans, Spectral theory and differential operators (Clarendon Press, Oxford, 1987).

16. A. Favini, Su una estensione del metodo d'interpolazione complesso, Rend. Sem. Mat. Univ. Padova 47 (1972), 243-298.

17. D. L. Fernandez, Interpolation of $2^{n}$ Banach spaces, Studia Math. 45 (1979), 175-201.

18. L. M. FERnÁndEZ-CABRERA, Compact operators between real interpolation spaces, Math. Inequal. Applic. 5 (2002), 283-289.

19. C. FoiAş AND J. L. Lions, Sur certains théorèmes d'interpolation, Acta Sci. Math. (Szeged) 22 (1961), 269-282.

20. S. Heinrich, Closed operator ideals and interpolation, J. Funct. Analysis 35 (1980), 397-411.

21. A. Pietsch, Operator ideals (North-Holland, Amsterdam, 1980).

22. M. ScheCHTER, Principles of functional analysis (American Mathematical Society, Providence, RI, 2002).

23. G. SpARR, Interpolation of several Banach spaces, Ann. Mat. Pura Appl. 99 (1974), 247316.

24. H. TRIEBEL, Interpolation theory, function spaces, differential operators (North-Holland, Amsterdam, 1978).

25. A. Yoshikawa, Sur la théorie d'espaces d'interpolation: les espaces de moyenne de plusieurs espaces de Banach, J. Fac. Sci. Univ. Tokyo 16 (1970), 407-468. 